\title{
System of Web-Based Electronic Medical Record
}

\author{
Lourdes M. Brasil ${ }^{1}$, Carolina G. Abreu ${ }^{2}$, Arlindo G. Vieira ${ }^{1}$, Maurício A. Machado ${ }^{1}$, Valfran S. \\ Almeida ${ }^{1}$, and Rodrigo M. Rodrigues ${ }^{3}$
}

${ }^{1}$ Catholic University of Brasília - Master's Program in Knowledge Management and Information Technology, SGAN 916, Modulo B, Campus II, Zip.: 70.790-160

Brasília, Brazil

lmb@ucb.br,arlindo@unimedcentro.com.br, mauricio@linkexpress.com.br, valfran.almeida@bsb.politec.com.br

${ }^{2}$ Catholic University of Brasília - Computer Science, SGAN 916, Modulo B, Campus II, Zip.: 70.790-160

Brasília, Brazil

abreu.carol@gmail.com

${ }^{3}$ Clinical of Cardiology, SHLSul 716 - Bloco E - Sala 604, Zip.: 70390-700

Brasília, Brazil

rodrigorodrigues59@gmail.com

\begin{abstract}
Nowadays, the information systems are considered a tool to make-decision support in several areas. One of the applications of this system could be in the development of a web-based Electronic Medical Record. The attention to standards, naming, accurate measuring and the system security in the sense of information privacy are fundamental elements in the development of a web-based electronic medical record. Therefore, based on the solidarity and maturity of web applications, this work presents a solution that could supply the construction of electronic medical records by the internet. Recently, in the Brazilian market there have been few successful initiatives. Taking this into account, this work proposes the use of proven software development methodologies. How a study case was used the angiology and vascular surgery. Currently the medical consultation processes of the angiology and vascular surgery specialties are operated manually. The final product provides automatization of these procedures.
\end{abstract}

Key-words: Electronic Medical Record, Information System, Angiology and vascular surgery.

\section{Introduction}

If there is something constant in the health industry is the change. The technology model for healthy care is in constant progress for new challenges are always being discovered. For this reason, the health product's industry looks ways to reduce costs and to introduce new advances.

The health industries are using the information technology to reduce expenses and to implement innovative and more efficient products. In the last to years there was an increase in the implementations of Electronic Medical Record (EMR) based in the information exchange and images documentation. Expert market consultants affirm that more than $60 \%$ of Health Institutions already have implemented or intend to implement this technology. This acceptance of the market is due to the possibility of simultaneous access, safe storage of the patient's clinical history, signal and image processing, as well multimedia information.

The use of computational resources can assist the medical practice, as much in diagnostic as in the analysis of the medical procedures. It is helpful on publishing results, gathering dispersed information, provide better quality service with a minor cost.

Due to it the next sections will be approached the aspects of standardization of an EMR, its development and a study case for the system validation. 


\section{Standards for the EMR of Patients for Storage and Representation of Information}

Some industrial sectors codify its products with the main objective to facilitate its control and distribution. As well as the products, there was the necessity to make the same in some services segments, as in the health area. For the sprouting of Medical Information Systems, the codification and the classification of clinical procedures had acquired important roles in Health Informatics.

Nowadays we can affirm that the classification and codification systems are the main components of electronic clinical registers for its capacity to keep organized and structuralized data. The classification systems have its importance in health for assist makingdecision, search and technological areas due to the great advantage to make possible the accurate information storage and exchange, allowing the communication between health institutions all over the world.

For better understanding of the standards of information storage and exchange, it is necessary to revise some concepts [6]:

Code - a system of words, letters or symbols, so that information can be stored or exchanged between different computer systems.

Nomenclature - A classified system of names.

Classification - Represent terms and concepts well organized, as well the relation between them.

\subsection{Standards}

The necessity of standards to regulate information is due to some factors [1]:

- The diversity of medical terms and sources (there is more than 150,000 medical concepts);

- The systems are built over different software and hardware platforms needing the standard by which all systems will communicate and exchange data;

- To make easier the search of data;

- For health statistics, epidemiology, clinical research and others specific purposes;

- To make possible the use of decision support systems and alert systems;

- To create portable systems.

The lack of standardization in the EMR provokes the data loss and makes impracticable many of the resources that could be offered, such as portability, clinical research, decision support systems, among others [1].

There are a large set of standards that are used in medical systems. There are vocabulary standards (ICD,
SUS Table, AMB, SNOMED, UMLS, etc), content standards (ABRAMGE), communication standards (HL7, X12, EDIFACT, XML, etc), images standards (DICOM), objects standard (CorbaMEd), for example.

According to Blois and Shortliffe [2], the standards used in the medical area can be classified:

- Identification: for patients (Social Security Number, in the United States; National Card of Health, in Brazil, etc.), for doctors (Register Number of the Medicine Council), etc;

- Communication; standard to exchange data between systems (HL7, X12, EDIFACT, XML, etc.);

- Concept and Structure: standardization of the clinical register (DATASUS, ABRAMGE, etc.);

- Clinical data representation (Codes): ICD, SNOMED, WHO, AMB, etc;

- Confidentiality, Security and Authentication (CEN, SBIS);

- Quality indicators, Guidelines (SBIS, GT Certification).

Some of these standards will be detailed according to the Brazilian scenario.

\subsubsection{International Classification of Diseases (ICD)}

The International Classification of Diseases (ICD) was idealized by the World Health Organization (WHO), in 1999, where ten versions were homologated until the current days [13]. The ICD is revised periodically by governmental agencies such as National Center of Health Statistics (NCHS) and Health Care Financing Administration (HCFA), these agencies only can incorporate changes in the classification of illnesses.

The ICD has become the international standard diagnostic classification for all general epidemiological and many health management purposes. These include the analysis of the general health situation of population groups and monitoring of the incidence and prevalence of diseases and other health problems in relation to other variables such as the characteristics and circumstances of the individuals affected [3].

It is used to classify diseases and other health problems recorded on many types of health and vital records including death certificates and hospital records. In addition to enabling the storage and retrieval of diagnostic information for clinical and epidemiological purposes, these records also provide the basis for the compilation of national mortality and morbidity statistics by WHO Member States.

Its main innovation was the use of a four characters alphanumeric code that consists of three numbers followed by a letter. With this new layout of codification it was possible to duplicate the universe of the set of codes if compared with the previous version. During years the 
ICD comes suffering constant alterations being that to each alteration a version is created. Therefore, in Brazil, for intermediary of the official document $\mathrm{n}^{\circ} 1,311$, September 12th of 1997, the Health Ministry defined the implantation of the ICD-10 in all the domestic territory.

\subsubsection{Health Level 7}

Health Level Seven (HL7) is one of several American National Standards Institute (ANSI) -accredited Standards Developing Organizations (SDO) operating in the healthcare arena. 'Level Seven' refers to the highest level of the International Organization for Standardization (ISO) communications model for Open Systems Interconnection (OSI) - the application level. The application level addresses definition of the data to be exchanged, the timing of the interchange, and the communication of certain errors to the application. The seventh level supports such functions as security checks, participant identification, availability checks, exchange mechanism negotiations and, most importantly, data exchange structuring.

HL7 is an international community of healthcare subject matter experts and information scientists collaborating to create standards for the exchange, management and integration of electronic healthcare information. HL7 promotes the use of such standards within and among healthcare organizations to increase the effectiveness and efficiency of healthcare delivery for the benefit of all. Health Level Seven's domain is clinical and administrative data [6].

The HL7 allows that different computational applications change excellent sets of medical, clinical and administrative information, in compliance with the Brazilian Legal System, all the users and producers of health information systems would have to produce minimum a set required of information.

HL7 most widely used protocol is the Application Protocol for Electronic Data Exchange in Healthcare Environments [5]. The HL7 presents support to different programming languages and operational systems, also enclosing the Internet. Moreover, it is one of the most used standards is EMR construction for its ample application - it creates interfaces for all the necessary specifications in a health organization, it has national and international recognition, the biggest centers of worldwide health use protocols HL7, and has relatively easy accessibility - its protocols quickly are implemented and with high responsibility for its members.

\subsubsection{World Health Organization}

World Health Organization (WHO) is the directing and coordinating authority for health within the United
Nations system. It is responsible for providing leadership on global health matters, shaping the health research agenda, setting norms and standards, articulating evidence-based policy options, providing technical support to countries and monitoring and assessing health trends [13].

The WHO contains important statistics and information on the world-wide health and serves as safe base in research. Evidence provides the foundation for setting priorities, defining strategies, and measuring results. WHO generates authoritative health information, in consultation with leading experts, to set norms and standards, articulate evidence-based policy options and monitor the evolving global heath situation [13].

\subsubsection{Brazilian Health System Database}

In Brazil the initiatives of the Brazilian Health System Database (DATASUS - Banco de Dados do Sistema Único de Saúde) are distinguished. It is an agency of the Health Ministry, responsible for the collection, processing and dissemination of the health information on Brazil, in standardizing the information in health in our country. A Brazilian example of standardization is the committee of Clinic Register Standardization (CRS) that it approved, through an opened process, the minimum set of data that an EMR must have. Moreover, a Document Type Definitions (DTD) corresponding to the structure of data in the SRC was elaborated using XML [4].

The Health Ministry, through the Health National Card, aims to identify the Brazilian citizen in the Health National System. It also launched a set of DTD recently, with the standardization for information exchange between the Health National Card systems [4].

It is of extreme importance that the EMR developed in Brazil have the capacity to communicate with the Brazilian Health System Database, due to its large national extension, so that in the future the information exchanges between the public system and the private system happen on an efficient form, consolidating the Information and Computer Science National Politics of in Health.

\subsubsection{Brazilian Society of Informatics in Health}

The Brazilian Society of Informatics in Health (SBIS Sociedade Brasileira de Informática em Saúde) aims to promote the development of all the aspects of the Health Information Technology. The tradition of the performance of the SBIS is marked by the accomplishment of national and international events, as congresses, symposiums, courses, seminaries and workshops [11].

The SBIS and the Federal Medical Council (CFM - Conselho Federal de Medicina) gathered to create rules 
for the EMR that appeared in the country and to take care of clinics and hospitals that would like to implement the EMR with security and control. They created the GT Certification.

The process of certification SBIS/CFM is destined to systems that capture, store and exchange health information identified, which means Local and Shareable Systems of Electronic Register, since that they fulfill the resolutions described in [9].

\section{STUDY CASE}

A solution suggestion of an EMR will be presented in which the health professional counts on a tool of aid to the patient's information management, allowing the remote access by a computer connected to the internet. Through the considered system it will be possible to store patient's information, on a trustworthy integrated form, making possible the remote access of any computer that possess access to the internet.

This study case it is a demonstration of a simple system that can be used by angiology and vascular surgery doctors. The data presented has been gotten from angiology and vascular surgeons doctors of the Clinical of Cardiology in Brasilia, Brazil.

Usually the lack of a system that allows the management of the patient's information can cause the following problems: Lack of standardization in the patient's record management process; Precarious storage of the records in paper; Difficulty in the localization of one determined record; Difficulty in sharing the patient's information points located remotely; Difficulties to establish safe politics of contingency in case that the environment where the paper patient's record is stored suffers damages as fires, overflows, robberies and etc; Little security in the access to paper records information; Errors in the diagnostic or procedures caused due to information or incomplete patient's data; Lack of control of the norms and standards defined for the government and medical associations in relation to EMR.

Due to variety of options that an Electronic Record system can provide, the system considered for the study case in question is destined only to the management of an EMR in Web environment. This EMR contains the necessary functionalities to get relevant patient information in an angiology and vascular surgery clinic. Any other necessities, such as images manipulation in medical examinations, will not be contemplated in this study case. This system was called System of Web-based Electronic Patient Record (SPW).

\subsection{SPW}

The SPW [12] was developed using as focus the medical clinics, therefore the biggest difficulty of the doctors is to offer an attendance to the patient in distinct doctor's offices, however having access the same data base with all the description of the patient since its first exam, allowing the doctor to have access to all the former information 24 hours per day, seven days of the week, in any place of the world.

The system is completely on Open Source software, as much to reduce the cost of development and, consequently, the final cost of the product, and to allow new research and innovations, since the community of free software is in constant evolution.

For the databases framework the Hibernate persistence was used [4], which allows the objectrelationary mapping and the transactions control, in a transparent form. It still allows that a change in XML will de refactored on the database.

Another framework that it was used in the system construction is the Struts. This framework is based on model MVC and possess many intrinsic resources. It makes the Web system construction easier, with validation resources, errors treatment, and form models, based on the standard Composite View.

The security of SPW had been defined with the use of the implementation of API JAAS supported for the server of Jboss applications [8]. This is a resource that also becomes transparent for the application the protection of the not authorized access and also is totally integrated with the necessities of the system. It allows users and profiles to access to the system.

For the system implemantation it was used a server with Linux Operational Service, MySQL DataBase 4.1 [10], J2SDK 5.0 [7] and a server for Jboss Application. The client systems can be executed from the car and open with all platform, it is necessary to the browser to process of information.

For the proposed study case three kinds of actors were identified: doctor, secretary and system management. Actors are elements that will interact with the system [12]:

a) Doctor: responsible for the records information management. There is no blocking access and has autonomy to decision taking. He also responds any patient question. The doctor should perform the following activities:

- Register patient health situation;

- Register patient biometry;

- Realize patient physical examination;

- Diagnosis of patient disease;

- Suggest or perform patient treatment; 
- Ask for medical exams;

- Generate reports, prescriptions, etc.

b) Secretary: responsible for patient identification management. He/She restricted access to specific system modules. He/She performs the following activities:

- Register patient ID information;

- Storage patient general information;

- Schedule control and patient's telephone contact.

c) System Management: responsible to manage system users' information.

On a bigger institution, such as hospitals, we can identify more actors (such management assistants, nurses, etc), but in this case it won't be necessary.

The flow of the patient attendance process begins with a patient appointment register. The secretary, after to effect the register of the patient data, creates a new appointment that appears in the doctor's schedule. The doctor takes care of the patient, effecting health conditions registers in the patient cadastre. He can also register examinations, diagnosis, prescriptions etc. Once the register is in the system, it cannot be modified. It is standed out that each event can be associated: none, one or more treatments or examinations. The information contained in the EMR are organized by way events that the doctor can have data access of the previous consultations.
For the system implementation, class diagrams were used. A class diagram is a representation of the structure and relations of the class that serve as model for objects that compose the system. A class abstracts a set of objects with similar characteristics. A class defines the behavior of its objects through methods and the possible states of these objects through attributes, which means a class describes the services provided for its objects and which information they can store. From the suggested model some system characteristics are verified. The system can store some patients. Which patient presents some attributes as name, address, telephone, sex, etc. A patient could have an appointment. One appointment has always a doctor's. An appointment can or not contains: Prescriptions, Examinations, Biometry, Physical Examination of Doppler Continuous Waves, Lymphatic Physical Examination, Physical Examination Pulses, etc. Figures 1 up to 6 shows some examples of the implementation`s SPW, version in Portuguese.

This is a simplified model to be used by angiology and vascular surgery doctors. SPW does not have the intention to take care of to all the necessities of the medical specialty of the angiology and vascular surgery areas, but to offer a possible implementation of an EMR, that can be functionalities added to the model.

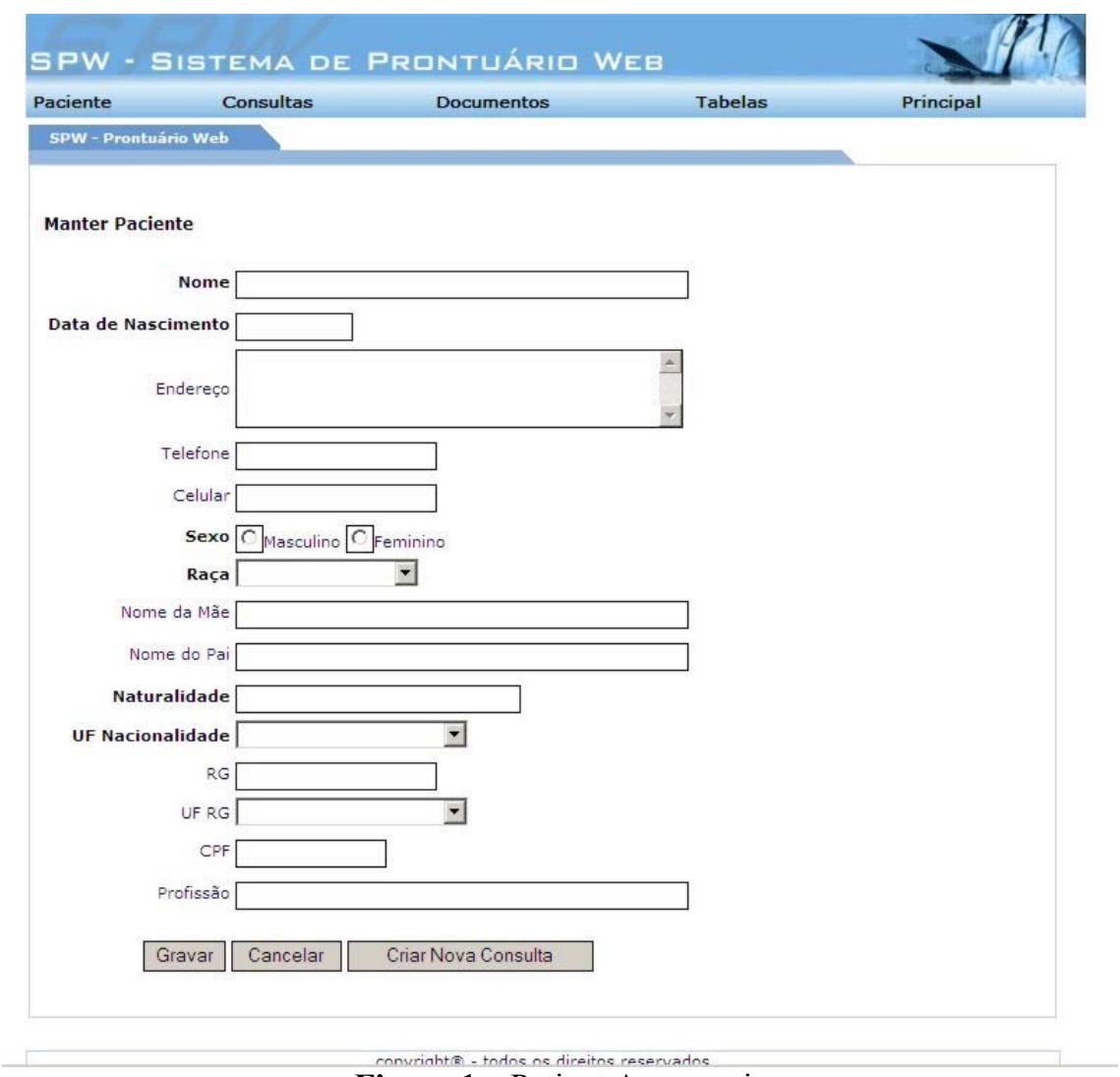

Figure 1 - Patient Anamnesis 


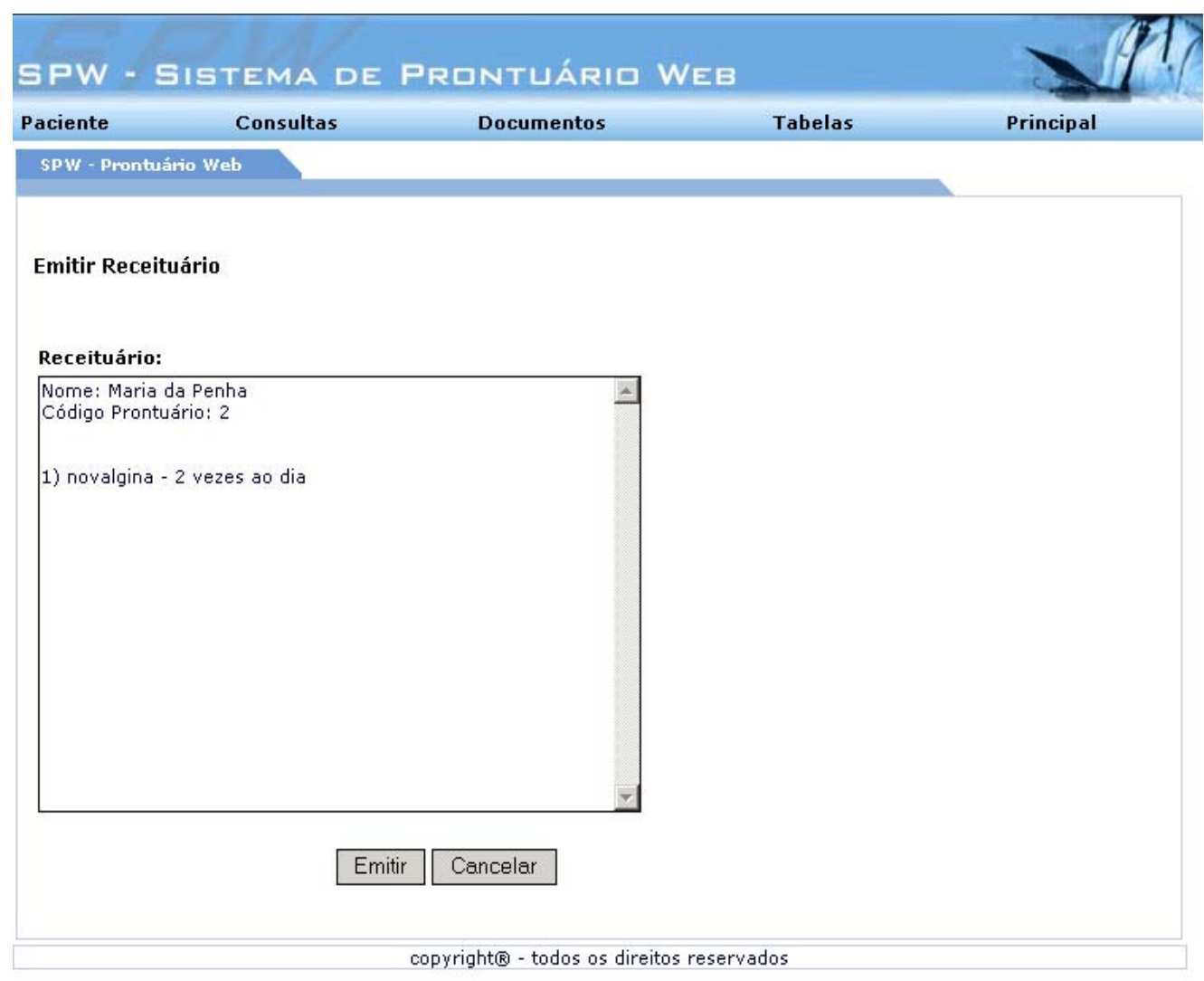

Figure 2 - Medical Fitness Certificate 


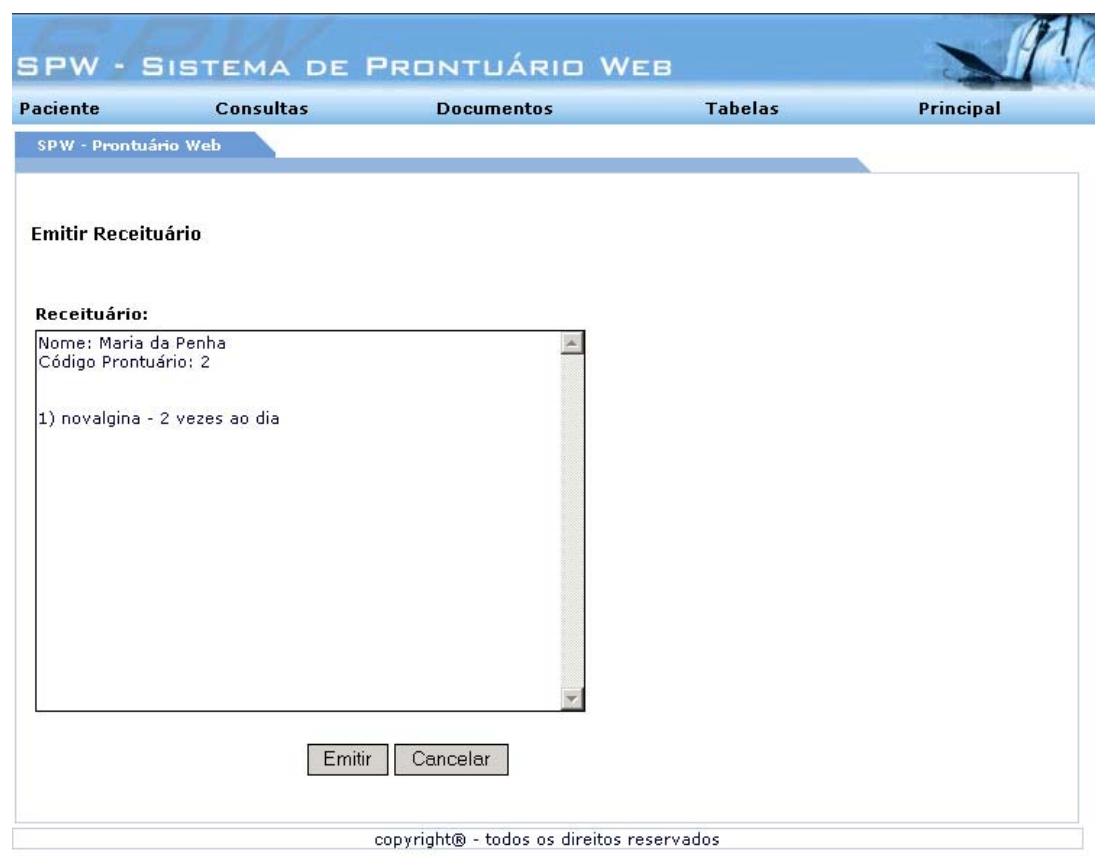

Figure 3 - Edit Assessment

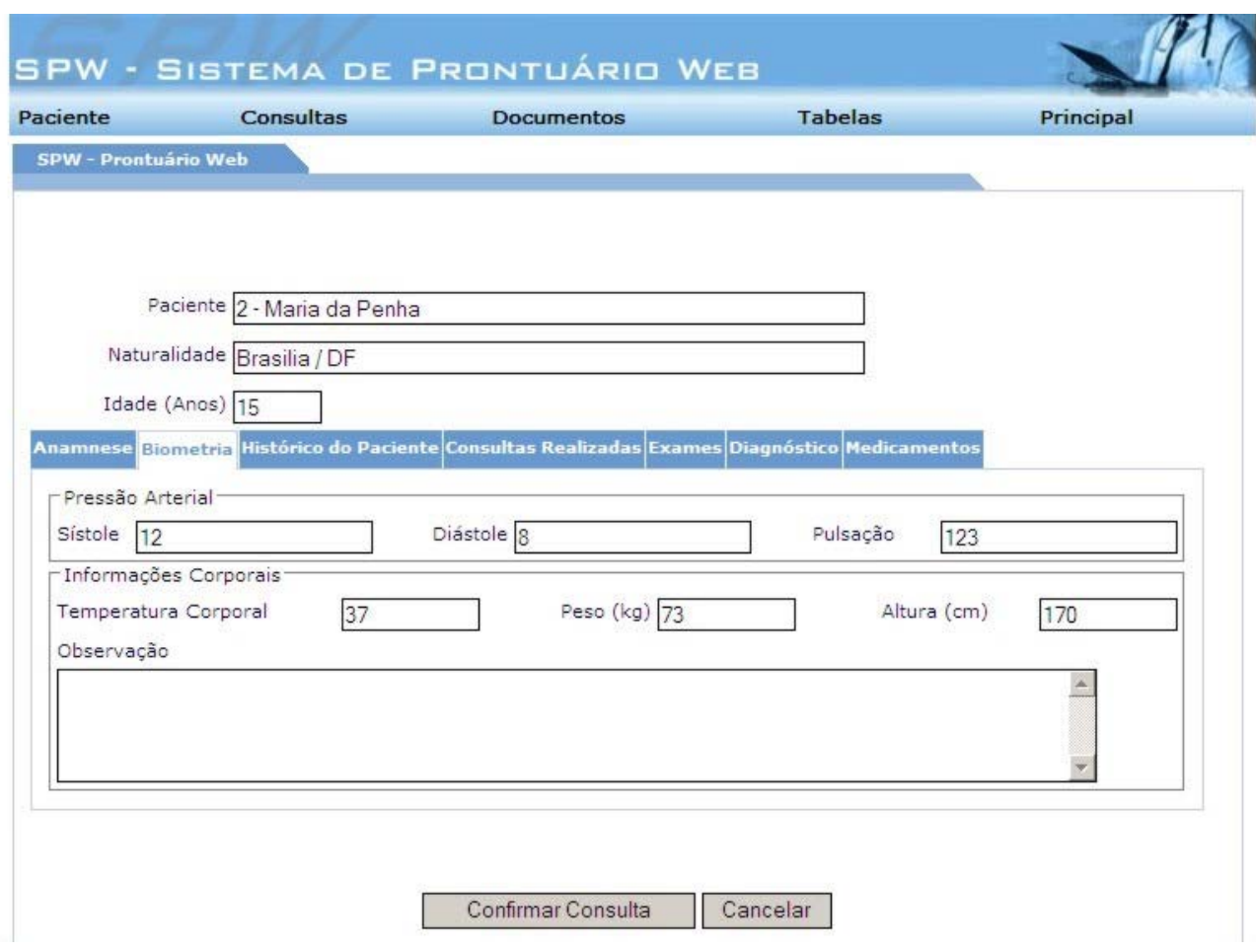


Figure 4 - Physical Examination and Tests Results

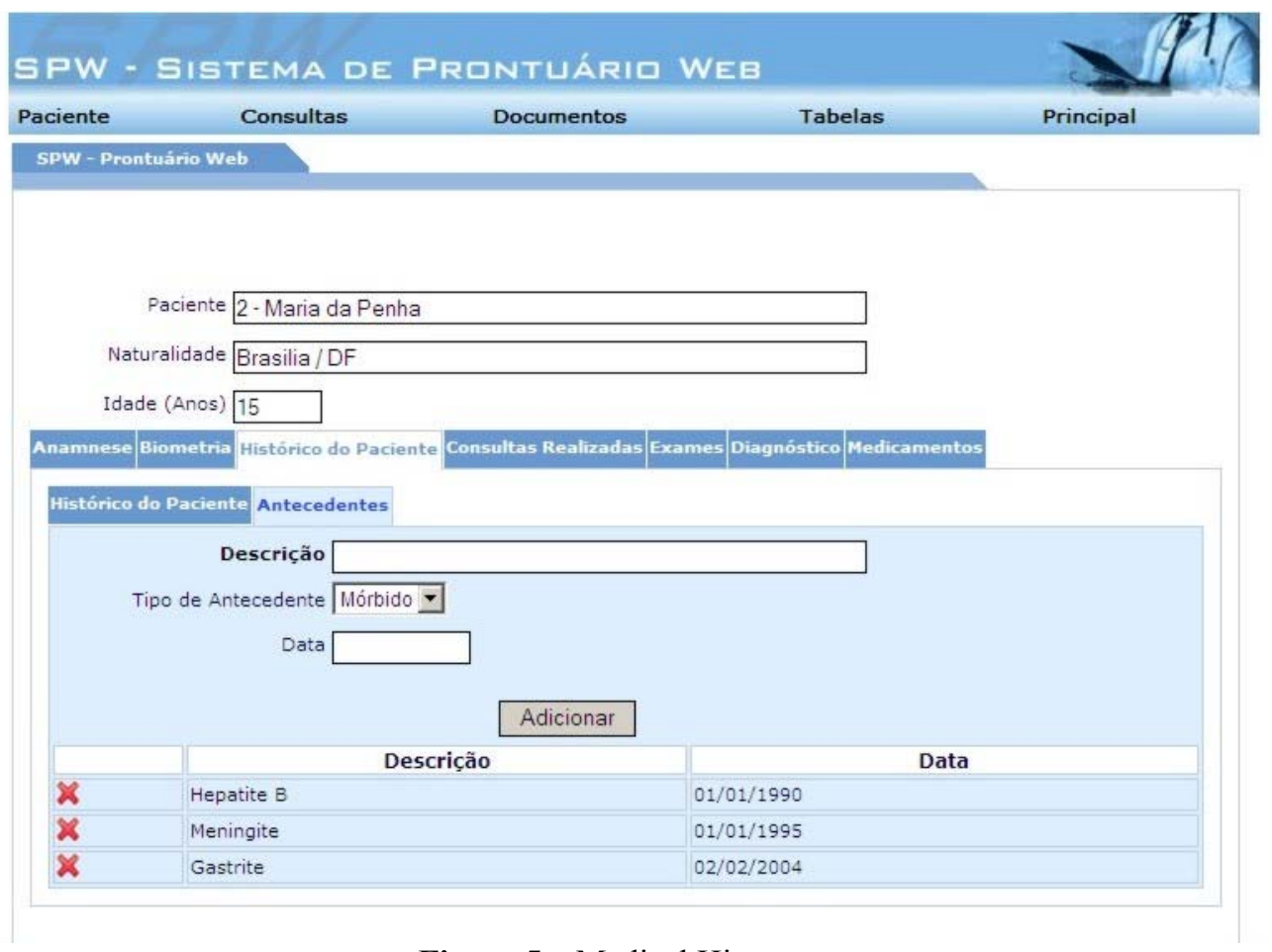

Figure 5 - Medical History 


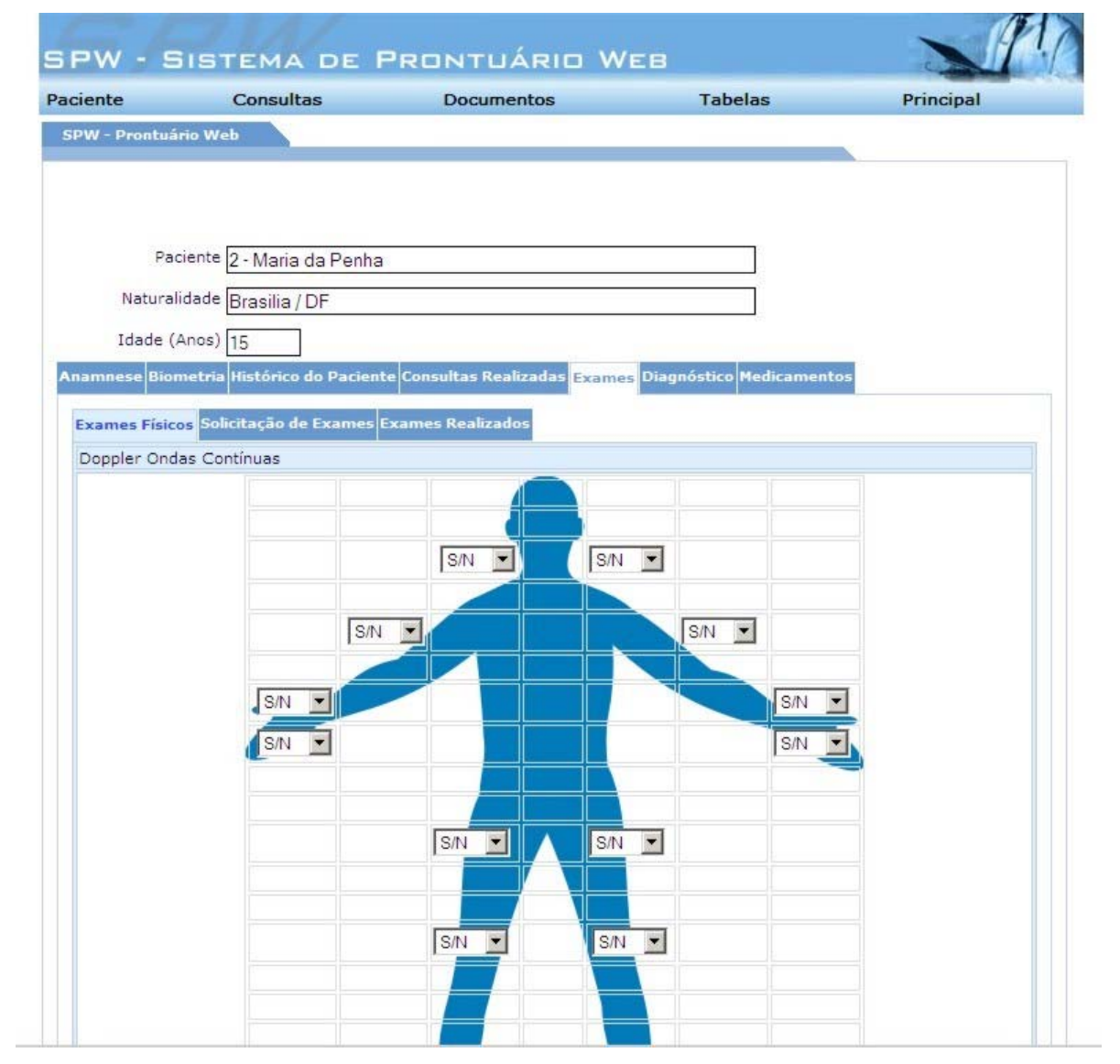

Figure 6 - Doppler Examination Interface in SPW

\section{Conclusions}

The SPW system implantation reached the following benefits: Optimized process record management; Structured organization of the record; Efficiency in the search of patient information record; Remote access to the patient information record; Guarantee of the patients information security; Adequacy to the norms, standards and procedures; Data legibility; Technician terms standardization and improvement in the quality of the patient treatment.

With the expansion and simplicity of the internet use, the applications web has become more common, e.g., why SPW offers web functionalities such as: Independence of locality for access; Information sharing; Security; Processing and storage; Simplicity in the use and for there is no need to install software in customers machines. With this, one became possible the creation and the development of a system that assisted medical professionals in the EMR management in medical clinics, making easy the information recovery, without harming its storage or same security. The patient who searches a treatment personalized and detailed can count on the web services to increase the quality of medical attendance in a medical clinic.

The SPW aimed to reach the maximum possible functionalities in the scope of the relation doctor-patient, changing the clinical appointment process. Using this system the clinical register patient data can be obtained on a centered, structured way. All data can be accessed by any computer hardwired to the internet.

The SPW aims to reach the maximum possible functionalities in the scope of the relation doctor-patient, making the clinical attendance process faster. From this system, the clinical register data can be gotten in a centered, structuralized and legible way, providing access to such information with any computer hardwired to the internet.

The doctor can also have access to the patient clinic history. For this reason he has access to all kinds of information such as previous diagnosis and prescriptions. 
Another system advantage is the printing of legible medical documents.

To build an EMR there are a set of aspects that must be considered, such as maximum medical specialty range, access control, if the system is easy to use, the interface is friendly, the standards protocols are being followed. Nowadays, the similar systems can only be used with few medical experts, enclosed in its origin institution. There is no way the patient clinic history is available for others health centers, making the communication among institution almost impossible. Lots of the nowadays EMR problems could be solved by using open standards such as HL7, DICOM, XML, etc. As long we keep focusing on a general health system and not on a solid EMR, we will keep finding difficulties in the different systems communication.

\section{References}

[1] ABREU, C., BRASIL, L. M., SILVA, R.R.J., LIMA, E.S. \& ALMEIDA, A.E.M. Levantamento de Dados para a Construção de um Prontuário Eletrônico do Paciente, Proceedings of the $V$ Workshop de Informática Médica (WIM2005), CD-ROM, Porto Alegre, Brazil, 2005.

[2] BLOIS, M. S. \& SHORTLIFFE E. H. Medical informatics: computer applications in health care. Publisher: Addison-Wesley Longman Publishing Co., Boston, 1990.

[3] CID. Classificação Internacional de Doenças. http://www.virtual.epm.br/material/tis/currmed/temas/med5/med5t12000/voc/cid/sld001.htm (May 10, 2006).

[4] DATASUS. Database SUS. http://www.datasus.gov.br (December 20, 2006).

[5] HL7. Health Level Seven - Data Model Development. http://www.hl7.org (June 8, 2007);

[6] ILHA, J.O. O Registro Clínico Computadorizado: Padronização e Codificação. Revista Informédica 1, 5, 1993, 5-8.

[7] J2SDK. Java Development Kit. http://www.java.sun (20/June/2006).

[8] JBOSS. Server of JBoss Application. http://www.jboss.com (10/June /2006).

[9] MANUAL. Manual de Requisitos de Segurança. Conteúdo e Funcionalidades para Sistemas de Registro Eletrônico em Saúde. Technical Report No. 20040219, 2004, p.70.

[10] MYSQL. Database MySql. http://www.mysql.com (20/November/2006).

[11] SBIS. Sociedade Brasileira de Informática em Saúde. http://www.sbis.org.br (July 6, 2006).
[12] VIEIRA, A., MACHADO, M. \& ALMEIDA, V.: SPW - Sistema de Prontuário Web. Monografia de Bacharelado em Ciência da Computação, UCB, Dez. 2005.

[13] WHO, World Health Organization Bridging the "Know-Do" Gap Meeting on Knowledge Translation in Global Health (Genebra, 2006). http://www.who.int (December 14, 2006).

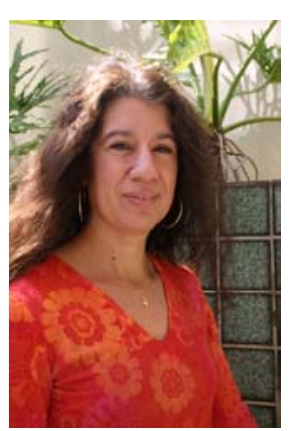

Lourdes Mattos Brasil received the B.Sc. degree in Electrical Engineering in 1989 from the Federal University of Santa Catarina, Brazil. In 1994, she received the M.Sc. degree and in 1999 she received the D.Sc. degree in Biomedical Engineering from the Federal University of Santa Catarina, Brazil. From 1997 until 1998, she developed researches in hybrid system by the Université Notre Dame de la Paix, Namur, Belgium. Since 2002, she is "Professor Adjunto" in the Catholic University of Brasília, Brazil, where she has been developing research projects and supervising graduate students in hybrid systems, artificial neural networks, pattern recognition, data-mining, virtual reality and so on in artificial intelligence. She has been entrepreneur from the Financer of Studies and Projects (FINEP) in Brazil, since 2005. She has also been director of Biomedical Engineering Course in the Catholic University of Brasília, Brazil, since 2004.

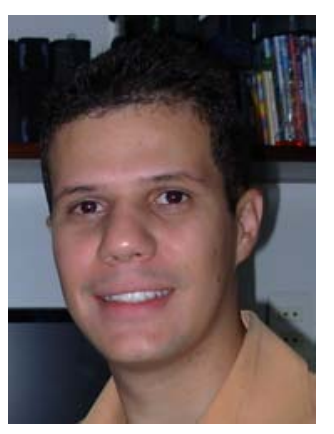

Valfran Santana de Almeida received the B.S.c. degree in Information Systems in 2005 from Catholic University of Brasilia. Since 2001 he has worked as a system programmer and system analyst in Politec Inc. where he been developed many systems for the principal banks and government of Brazil, using Java and JEE technologies. His final project was an Electronic Medical Record which has been served as an Angiology and Vascular Surgery specialty since 2005. Actually, this EMR is being used as a base for other systems in the medical area. 


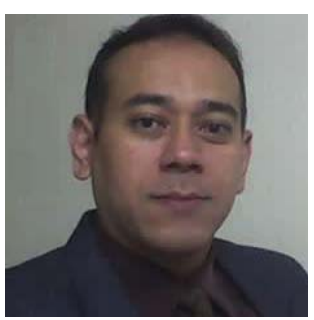

Maurício Abe Machado, received the B.Sc. degree in Information Systems in 2005 from the Catholic University of Brasília, Brazil, as well he received the title of expert in software engineering, Brazil, in 2007. Since 2003, he has working with development of medical softwares, using Java and J2EE. He works as System Analyst in the Unimed Centro-Oeste and Tocantins, Brazil, where he develops Java projects and Project Manager Officer member. His final project was an Electronic Medical Record which has been served as an Angiology and Vascular Surgery specialty since 2005.

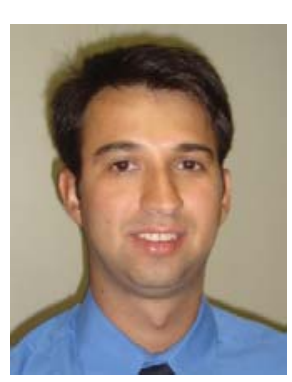

Arlindo Gonçalves Vieira Filho received the B.Sc. degree in Information Systems in 2005 from the Catholic University of Brasília, Brazil, as well he received the title of expert in software engineering, Brazil, in 2007. Since 2003, he has been working in the Center for Informatics at Unimed CentroOeste e Tocantins, Brazil, where he has been developing general systems for the medical area and information systems, using Java and J2EE. His final project was an Electronic Medical Record which has been served as an Angiology and Vascular Surgery specialty since 2005 .

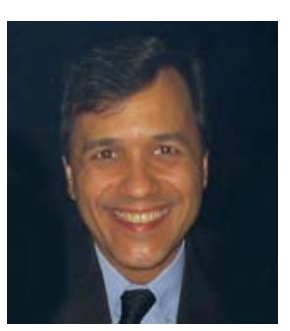

Rodrigo Macedo Rodrigues received the M.D. degree in Medical School at the Federal University of Uberlandia, in 1984. He concluded the Medical Residency in Angiology and Vascular Surgery in the Brasilia's Armed Forces Hospital in 1988. Since 1989 he has been working as physician staff of the Health's Secretariat of the Federal District. He had already occupied the Regional Secretary and Regional President of the Angiology and Vascular Surgery Brazilian Society positions, in 1997 and 1999, respectively.

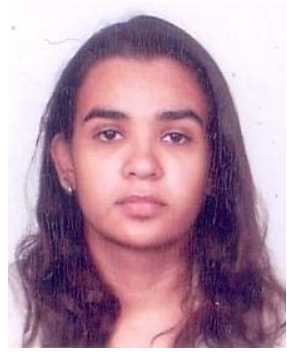

Carolina Gonçalves Abreu is a student in the B.Sc. degree in Computer Science at the University Catholic of Brasilia, Brazil and a student in the B. Sc. degree in Biology at the University of Brasília. Since 2004 she has been working in research projects in Virtual Reality, 3D Modelling, Knowledge Representation and Intelligent Agents. She has been a trainee from the Financer of Studies and Projects (FINEP) in Brazil, since 2005. One of her projects was an Electronic Medical Record (EMR) which has been used as an Angiology and Vascular Surgery Expert. Actually, this EMR is being used as a base for other systems in the medical area. 